\title{
Coenurus serialis in Northeastern Iran: A Probable Danger to Human
}

\author{
Amin Moshiri, ${ }^{1}$ Seyed Aliakbar Shamsian, ${ }^{2}$ Fariba Berenji, ${ }^{2}$ Abbas Jadidoleslami, ${ }^{2}$ and Elham \\ Moghaddas," \\ ${ }^{1}$ Department of Pathobiology, School of Veterinary Medicine, Ferdowsi University of Mashhad, Mashhad, Iran \\ ${ }^{2}$ Department of Parasitology and Mycology, Faculty of Medicine, Mashhad University of Medical Sciences, Mashhad, Iran \\ "Corresponding author: Elham Moghaddas, PhD, Department of Parasitology and Mycology, Faculty of Medicine, Mashhad University of Medical Sciences, Mashhad, Iran. \\ Tel/Fax: +98-5138002401-3, E-mail: moghaddase@mums.ac.ir
}

Received 2017 May 14; Accepted 2017 September 19.

\begin{abstract}
Background: Coenurosis is a zoonotic disease caused by the larval stage of Taenia multiceps or Taenia serialis species.

Objectives: The current study aimed at reporting the entity of Coenurus serialis in Iran.

Methods: The five dead rabbits found in Sarakhs city, Northeastern Iran, were inspection carefully. Coenurus serialis cysts recovered from their muscles were inoculated orally into a four-month-old Spitz/Terrier dog.

Results: Based on the current study, 40\% of the inspected rabbits in Northeastern Iran had C. serialis. The eggs appeared in the feces 58 days post-inoculation (dpi). Cestodes length was $15-25 \mathrm{~cm}$ and the total number of scolex hooks was $30 \pm 1$. The mean length of large and small hooks was 140 and $90 \mu \mathrm{m}$, respectively.

Discussion: Taenia multiceps and T. serialis in dogs were morphologically indistinguishable in the current study. The disease exists in Northeastern Iran in animals and is the potential danger as a zoonotic disease. The current study established the first experimental infection of C. serialis in Iran. Neurosurgeons should consider the probability of the disease in this region.
\end{abstract}

Keywords: Dog, Rabbit, Iran, Experimental Infection, Taenia serialis

\section{Background}

Taenia serialis (Taenia antartica) is already recorded in Europe, Africa, and North America (1). Canids including wolves, foxes, dogs, and coyotes are the definitive hosts (2); whereas, lagomorphs including both hares and rabbits are the usual intermediate hosts (3). The infections are also found in cats, humans, baboons, mandrill, squirrels, and other rodents (4). The intermediate hosts including humans are infected by eggs ingestion of dog's feces. Coenurus serialis (the larval phase of the T. serialis) settle in central nervous system (CNS). Six human cases, also seven cases of fatal cerebral coenurosis in cats were reported from America (5). One breast cyst of $T$. serialis larvae was detected in a 38-year-old Caucasian female. Cysts in human were presented in the CNS, muscles, and subcutaneous tissue (6). Coenurus serialis cysts in human are rare and only about 100 cases ( $C$. multiceps and C. serialis) were recorded until 2008 (7) with the most cases from Africa (8). In rabbits, most of the Coenuri are found in lumbar and hind legs muscles, rarely in jaw muscles (9).

\section{Objectives}

The current report describes the presence of coenurus, believed to be $T$. serialis, in the muscles of wild rabbits and provides the description of coenurus experimental infection in a dog. There was no published data of the prevalence of this parasite in Iran. The current study aimed at reporting the existence of these zoonotic parasites in Northeastern Iran and describing the characteristics of $T$. serialis in the definitive host in comparison with those of T. multiceps.

\section{Methods}

\subsection{Wild Rabbit}

Sarakhs geographic coordinate is $36^{\circ} 32^{\prime} 42^{\prime \prime} \mathrm{N}$ $61^{\circ} 09^{\prime} 28^{\prime \prime} \mathrm{E}$ with cold winters and warm-dry summers influenced by Gharaghoroom Desert. It is located in Khorasan province in Northeastern Iran (Figure 1). In 2015, five dead rabbits were found during three weeks. All the muscles were inspected carefully. 


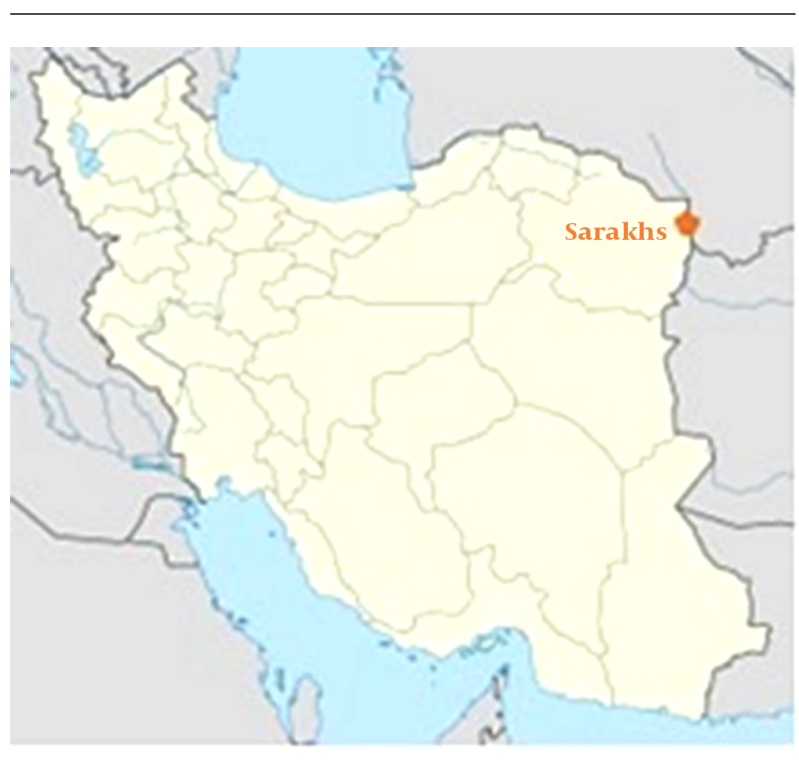

Figure 1. Location of hunting the rabbits infected With Coenurus serialis in Northeast of Iran

\subsection{Experimental Infection of a Dog with Coenurus serialis}

A cyst with about 40 scolices was obtained from the hind leg of a rabbit and was fed to a 4-month-old Spitz/Terrier dog. The puppy received oral administration of praziquantel tablets. Its fecal samples were examined both with sodium chloride floatation and sedimentation techniques to ensure that it was free from enteric parasite especially cestodes. The subject was euthanized using intravenous barbiturate overdose and was handled to necropsy hall with excellent hygienic condition. The intestine was opened longitudinally and the adult cestodes were collected for further investigations (Figure 2). After relaxation, they were compressed between two glass slides and fixed in 10\% formalin for at least 24 hours for further processing and staining with alum carmine stain. A calibrated eyepiece micrometer was used to measure the followings: the length of adult worms, the length of mature and gravid segment and the number and the length of large and small hooks. All the measurements were repeated three times by a parasitologist.

\subsection{Ethics}

All ethical standards including nutrition and good maintenance of dogs during experimental infection were respected.

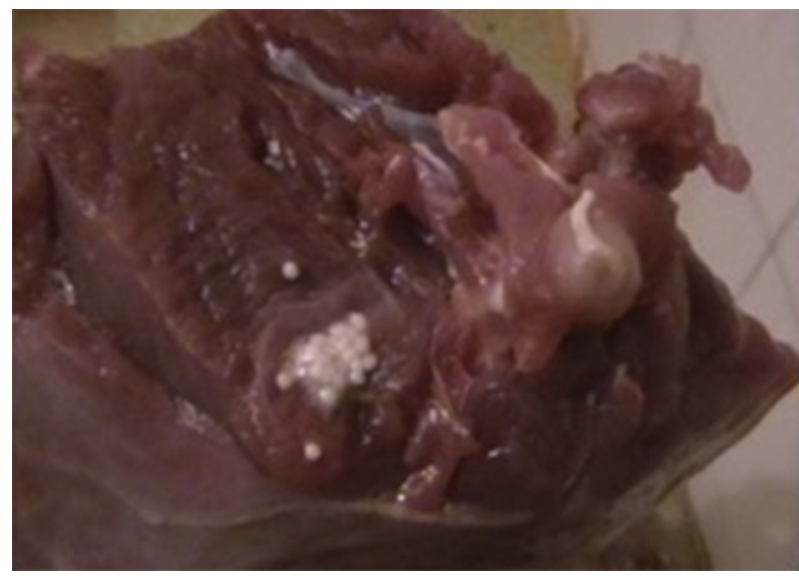

Figure 2. Coenurus serialis in muscle of rabbit

\section{Results}

\subsection{Wild Rabbit}

In the mentioned region in 2015 , two of five (40\%) dead wild rabbits were infected with four cysts of Coenurus serialis. One cyst was recovered from the lumbar muscle and three others from hind legs. The hind leg cysts were about $2 \mathrm{~mm}$ in diameter and the lumbar muscles cyst was $5.5 \mathrm{~mm}$ (Figure 3). The cyst wall was thin and individual protoscolices were above $0.5 \mathrm{~mm}$ long; the opaque-white cysts were distributed in close linear arrangements serially with 6 40 protoscolices. Microscopically, the scolex showed four cup-shaped suckers and the rostellum similar to other typical taeniid hooks was arranged in double row (large and small).

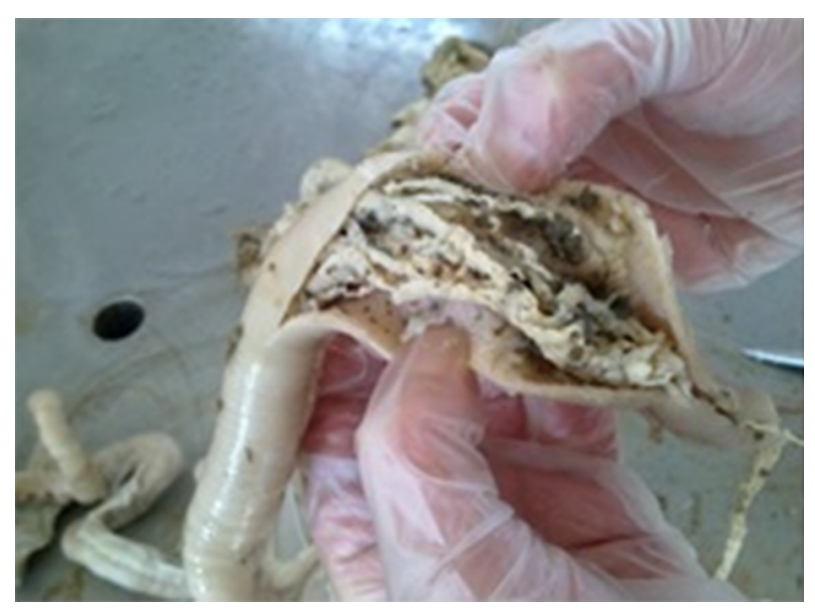

Figure 3. Taenia serialis in jejunum of dog infected experimentally with Coenurus serialis 


\subsection{Experimental Infection of a Dog with Coenurus serialis}

The experimental infection of the dog with C. serialis revealed that the first gravid segment of $T$. serialis was shed after 58 days post-infestation. Thirty-one adult worms were collected from small intestine of the subject; mostly attached to jejunum. No specific abdominal symptoms such as diarrhea or constipation were observed in the subject. The worms were whitish in color and $15-25 \mathrm{~cm}$ in length. The total number of hooks in each scolex was $30 \pm 1$. The mean length of large and small hooks was 140 and $90 \mu \mathrm{m}$, respectively. The mature segments were almost square in shape and measured $3.7 \mathrm{~mm}$ long and $2.5 \mathrm{~mm}$ wide on average and also contained a single set of genital organs. Gravid segments were usually longer; they were broad and measured $4.6 \mathrm{~mm}$ long and $2.2 \mathrm{~mm}$ wide. The number of lateral uterine branches ranged 21 to 23 on each side (Figure 4).

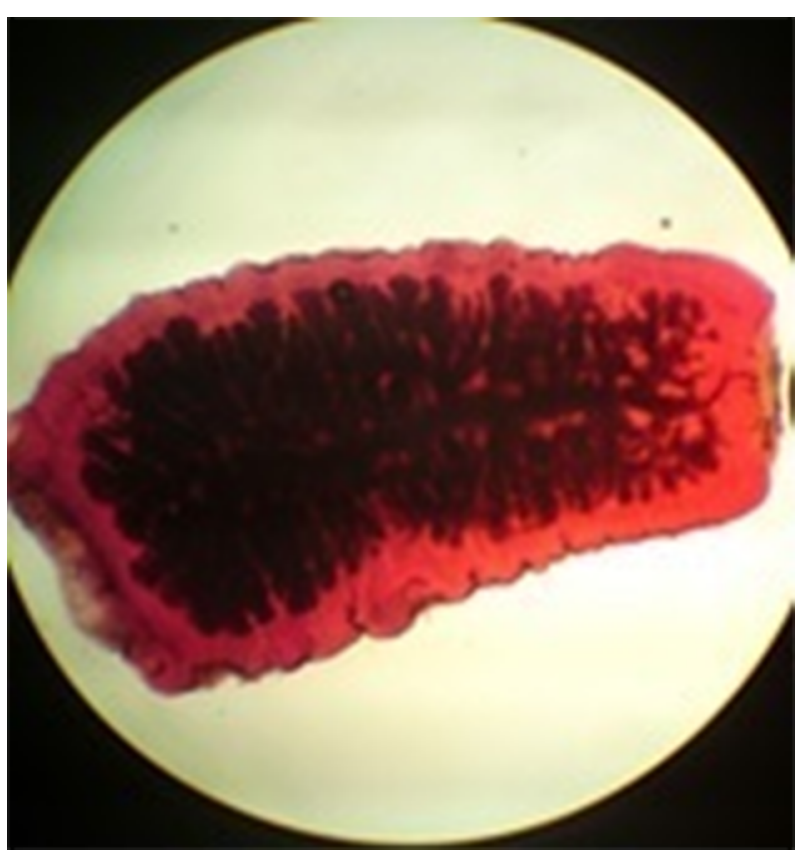

Figure 4. Gravid segment of Taenia serialis in dog intestine

\section{Discussion}

About 150 cases of coenurosis in humans were reported mostly in Africa, the United Kingdom, France, North America, and the Middle-East $(2,7)$.

In Europe and the USA, this parasite is very occasionally reported as the cause of subcutaneous cysts in humans (10) and in CNS of cats (5). In Eastern Europe, it is a known parasite among wolves (11). Experimental infections in rodents produced CNS involvement (12). The life cycle of $T$. serialis is completed through the ingestion of the rabbit meat by carnivores.

It is difficult to morphologically distinguish $C$. serialis from C. cerebralis, that is a wide spread parasite (13). Schneider-Crease molecular technique was used to diagnose this cyst in gelada since morphological approaches produced inconsistent results (14). It was reported that experimental infection in carnivores is critical. It is thought that they were best differentiated by transmission to dogs whereby the adult stages can be identified (15). Sheep and rabbits are T. multiceps and T. serialis intermediate hosts, respectively, while C. serialis is not recorded in sheep yet (16). In the current study, the mean length of large and small hooks was 140 and $90 \mu \mathrm{m}$, respectively, similar to those of T. multiceps. Mean \pm standard deviation (SD) of total length of the large and small hooks in T. multiceps was 120 - 195 and 73 - 150, respectively, in the literature (17). Each species had many protoscolices clustered together, usually in rows in C. serialis (18); hence, two species (T. serialis and T. multiceps) are considered as one genus by some authors (19). Other authors distinguished them on morphological grounds. In the current study, lengths and number of hooks and hooklets, and the number of branches of uterine were in the same range between the two species. Therefore, these criteria cannot be used to diagnose the two species. Other factors such as cirrus length and width as well as size and number of testes should be used to differentiate T. multiceps from T. serialis. According to the current study, shape and arrangement of protoscoleces inside the cyst is a simpler detector for the diagnosis of parasite species.

Yamashita et al. and Soulsby reported numerous scolices form radiating rows and daughter cysts in $C$. serialis cysts, but not in C. cerebralis (20); while in the current study, none of the cysts had any daughter cysts. In another study, the only morphological difference between $C$. cerebralis and $C$. serialis was scolex number of cysts as in $C$. cerebralis 500 to 700 in non-linear arrangement, and C. serialis 400 - 700 distributed in radial lines. The result of this report was in agreement with those of the current study about the liner-scolex shape in cysts, but at variance with scolex number of cysts.

In this regard, T. serialis was obtained during the experimental infection of the $\operatorname{dog}$ by $C$. serialis of wild rabbit, but it was not differentiated from $T$. multiceps according to usual morphological indicators. The current study results revealed that the prepatent period of this parasite was 58 days. A study declared the appearance of the eggs in feces 45 - 57 days post-infection (21). The current study result was in agreement with those of other works on prepatent period of Taeniidae family. Khorasan province is endemic for 
human hydatid cyst. In one study in this area, 1,342 cases of hydatidosis were recorded during 10 years (22). But the report confirmed that coenurosis can be a CNS cyst in human in the Northeastern Iran. Brain and nervous system surgeons in this region should consider three parasitic diseases: hydatid cyst, C. multiceps, and C. serialis. According to the study results, it is believed that cerebral coenurosis in the Northeast of Iran could be caused by $T$. serialis. These cysts are distinguished by different arrangements of protoscolices inside the cyst easily.

\subsection{Conclusions}

The current study was the first report of $C$. serialis in rabbit in Iran. In this regard, no data are documented about the existence of this parasite in humans. Clearly, further research is needed to predict and control the spread of the disease, especially among rural people and shepherds, due to the lack of hygiene education and the close contact with dogs. Results of the current study showed that diagnosis of cysts is easier than adult parasite; therefore, surgeons can differentiate this kind of cyst during the surgery.

\section{Acknowledgments}

Authors expressed their sincere thanks to Mashhad University of Medical Sciences, Iran for providing laboratory materials and equipment for the study.

\section{Footnotes}

Authors' Contribution: Amin Moshiri: sample collection; Elham Moghaddas and Abbas Jadidoleslami: drafting and revising the manuscript; Fariba Berenji: revision of the manuscript. All the authors read and approved the final version of the manuscript.

Conflict of Interests: The authors declared no conflict of interest.

\section{References}

1. Zammarchi L, Strohmeyer M, Bartalesi F, Bruno E, Munoz J, Buonfrate D, et al. Epidemiology and management of cysticercosis and Taenia solium taeniasis in Europe, systematic review 1990-2011. PLoS One. 2013;8(7). e69537. doi: 10.1371/journal.pone.0069537. [PubMed: 23922733].

2. Segovia JM, Torres J, Miquel J, Llaneza L, Feliu C. Helminths in the wolf, Canis lupus, from north-western Spain. J Helminthol. 2001;75(2):18392. [PubMed: 11520444].

3. Benger A, Rennie RP, Roberts JT, Thornley JH, Scholten T. A human coenurus infection in Canada. Am J Trop Med Hyg. 1981;30(3):638-44. [PubMed: 7196161].

4. O'Reilly A, McCowan C, Hardman C, Stanley R. Taenia serialis causing exophthalmos in a pet rabbit. Vet Ophthalmol. 2002;5(3):227-30. [PubMed: 12236877].
5. Conboy G. Cestodes of dogs and cats in North America. Vet Clin North Am Small Anim Pract. 2009;39(6):1075-90. vi. doi: 10.1016/j.cvsm.2009.06.005. [PubMed: 19932364].

6. Lescano AG, Zunt J. Other cestodes: sparganosis, coenurosis and Taenia crassiceps cysticercosis. Handb Clin Neurol. 2013;114:335-45. doi: 10.1016/B978-0-444-53490-3.00027-3. [PubMed: 23829923].

7. Benifla M, Barrelly R, Shelef I, El-On J, Cohen A, Cagnano E. Huge hemispheric intraparenchymal cyst caused by Taenia multiceps in a child. Case report. J Neurosurg. 2007;107(6 Suppl):511-4. doi: 10.3171/PED07/12/511. [PubMed: 18154024].

8. Nakao M, Lavikainen A, Iwaki T, Haukisalmi V, Konyaev S, Oku Y, et al. Molecular phylogeny of the genus Taenia (Cestoda: Taeniidae): proposals for the resurrection of Hydatigera Lamarck, 1816 and the creation of a new genus Versteria. Int J Parasitol. 2013;43(6):427-37. doi: 10.1016/j.ijpara.2012.11.014. [PubMed: 23428901].

9. Bethell F, Truszkowska A. Taenia serialis in a domestic rabbit. Vet Rec. 2010;166(9):282. doi:10.1136/vr.c1087. [PubMed: 20190227].

10. Ing MB, Schantz PM, Turner JA. Human coenurosis in North America: case reports and review. Clin Infect Dis.1998;27(3):519-23. [PubMed: 9770151].

11. Guerra D, Armua-Fernandez MT, Silva M, Bravo I, Santos N, Deplazes $\mathrm{P}$, et al. Taeniid species of the Iberian wolf (Canis lupus signatus) in Portugal with special focus on Echinococcus spp. Int J Parasitol Parasites Wildl. 2013;2:50-3. doi: 10.1016/j.ijppaw.2012.11.007. [PubMed: 24533315].

12. Nakao M, Yanagida T, Okamoto M, Knapp J, Nkouawa A, Sako Y, et al. State-of-the-art Echinococcus and Taenia: phylogenetic taxonomy of human-pathogenic tapeworms and its application to molecular diagnosis. Infect Genet Evol. 2010;10(4):444-52. doi: 10.1016/j.meegid.2010.01.011. [PubMed: 20132907].

13. Hosts-Taeniasis. Transmission and Life Cycle. The Center for Food Security and Public Health; 2005.

14. Schneider-Crease IA, Snyder-Mackler N, Jarvey JC, Bergman TJ. Molecular identification of Taenia serialis coenurosis in a wild Ethiopian gelada (Theropithecus gelada). Vet Parasitol. 2013;198(1-2):240-3. doi: 10.1016/j.vetpar.2013.08.015. [PubMed: 24050944].

15. Christodoulopoulos G, Kassab A, Theodoropoulos G. Characteristics of non-cerebral coenurosis in tropical goats. Vet Parasitol. 2015;211(34):216-22. doi: 10.1016/j.vetpar.2015.05.020. [PubMed: 26073108].

16. Oge H, Oge S, Gonenc B, Ozbakis G, Asti C. Coenurosis in the lumbar region of a goat: a case report. Veterinarni Medicina. 2012;57(No. 6):30813. doi: 10.17221/6018-vetmed.

17. Rostami S, Beech RN, Salavati R, Baneshi MR, Kamyabi H, Harandi MF Morphometric Analysis of Larval Rostellar Hooks in Taenia multiceps of Sheep in Iran and Its Association with Mitochondrial Gene Variability. Iran J Parasitol. 2013;8(4):579-85. [PubMed: 25516739].

18. Godara R, Borah MK, Sharma RL, Jangir BL. Caprine coenurosis with special reference to hepatic coenurosis. Comparat Clin Pathol. 2010;20(3):277-80. doi: 10.1007/s00580-010-1108-0.

19. Loos-Frank B. An up-date of Verster's (1969) 'Taxonomic revision of the genus Taenia Linnaeus' (Cestoda) in table format. Syst Parasitol. 2000;45(3):155-83. [PubMed: 10768761].

20. Monsang SW, Singh MN, Kumar M, Roy J, Pal SK, Singh WR. Diag nosis and surgical management of non-cerebral coenurosis (Taenia gaigeri) in local Black Bengal goat of Tripura: report of six cases. J Parasit Dis. 2016;40(3):914-8. doi: 10.1007/s12639-014-0604-6. [PubMed: 27605809].

21. Torgerson PR, Deplazes P. Echinococcosis: diagnosis and diagnostic interpretation in population studies. Trends Parasitol.2009;25(4):16470. doi: 10.1016/j.pt.2008.12.008. [PubMed: 19269248].

22. Andalib Aliabadi Z, Berenji F, Fata A, Jarahi L. Human Hydatidosis/Echinococosis in North Eastern Iran from 2003-2012. Iran J Parasitol. 2015;10(4):658-62. [PubMed: 26811735]. 